\title{
ON THE NON-LINEAR VIBRATION PROBLEM OF THE ELASTIC STRING*
}

\author{
BY \\ G. F. CARRIER \\ Harvard University
}

1. Introduction. It is well known that the classical linearized analysis of the vibrating string can lead to results which are reasonably accurate only when the minimum (rest position) tension and the displacements are of such magnitude that the relative change in tension during the motion is small. The following analysis of the free vibrations of the string with fixed ends leads to a solution of the problem which adequately describes those motions for which the changes in tension are not small. The perturbation method is adopted, using as a parameter a quantity which is essentially the amplitude of the motion. The periodic motions arising from initial sinusoidal deformations

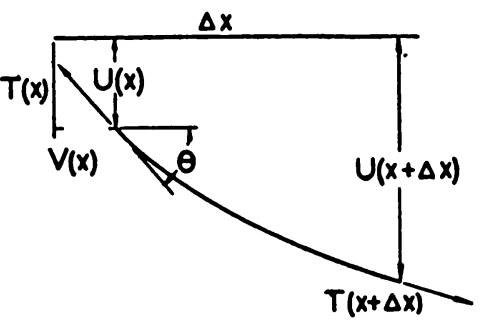

FIG. 1. Displaced element of string. are closely approximated in closed form. The method is applied to motions not restricted to a single plane and finally the exact solution for the transmission of a localized deformation is indicated.

2. The equations of motion. The equations of dynamic equilibrium of an element of the string, deformed into a plane curve as shown in Fig. 1, are

$$
\frac{\partial}{\partial x}[T \sin \theta]=\rho A \frac{\partial^{2} u}{\partial t^{2}}, \quad \frac{\partial}{\partial x}[T \cos \theta]=\rho A \frac{\partial^{2} v}{\partial t^{2}},
$$

where $\rho$ denotes the mass per unit volume, $A$ the cross-sectional area of the string in the rest position, and $\theta=\operatorname{arc} \tan \left[u^{\prime} /\left(1+v^{\prime}\right)\right]$, the primes indicating differentiation with respect to $x$. The condition of fixed ends implies that,

$$
\int_{0}^{l} v^{\prime} d x=0 \text { for all } t
$$

The stress-strain relation of the string is assumed in the form,

$$
T-T_{0}=E A\left\{\left[\left(1+v^{\prime}\right)^{2}+\left(u^{\prime}\right)^{2}\right]^{1 / 2}-1\right\},
$$

where $T_{0}$ is the tension in the rest position and $E$ is a constant characteristic of the string material. The following dimensionless quantities are introduced to simplify the algebraic work

$$
\alpha^{2}=\frac{T_{0}}{E A}, \quad \tau=\frac{T-T_{0}}{T_{0}}, \quad \xi=\frac{\pi x}{l}, \quad \eta=\frac{\pi}{l}\left(\frac{T_{0}}{\rho A}\right)^{1 / 2} t .
$$

After differentiating Eqs. (1) with respect to $x$, setting

* Received Jan. 3, 1945. 


$$
\begin{aligned}
& \sin \theta=\frac{u^{\prime}}{\left[\left(u^{\prime}\right)^{2}+\left(1+v^{\prime}\right)^{2}\right]^{1 / 2}}=\frac{u^{\prime}}{1+\alpha^{2} \tau}=\varphi, \\
& \cos \theta=\left(1-\varphi^{2}\right)^{1 / 2},
\end{aligned}
$$

and eliminating $v^{\prime}$ between Eqs. (1) and (2), we obtain

$$
\begin{aligned}
\frac{\partial^{2}}{\partial \xi^{2}}[(1+\tau) \varphi] & =\frac{\partial^{2}}{\partial \eta^{2}}\left[\left(1+\alpha^{2} \tau\right) \varphi\right], \\
\frac{\partial^{2}}{\partial \xi^{2}}\left[(1+\tau)\left(1-\varphi^{2}\right)^{1 / 2}\right] & =\frac{\partial^{2}}{\partial \eta^{2}}\left[\left(1+\alpha^{2} \tau\right)\left(1-\varphi^{2}\right)^{1 / 2}\right] \\
\int_{0}^{\pi}\left(1+\alpha^{2} \tau\right)\left(1-\varphi^{2}\right)^{1 / 2} d \xi & =\pi
\end{aligned}
$$

These equations rigorously define the motion of the string which is acted on by on external forces.

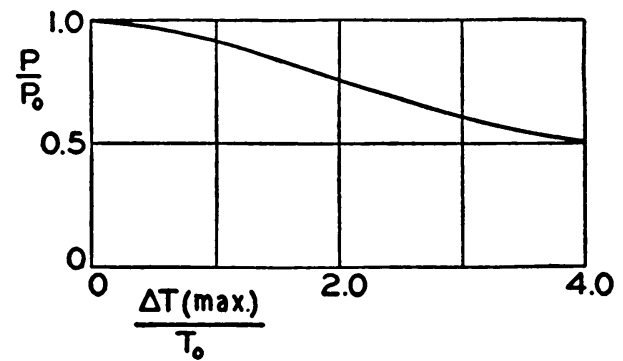

FIG. 2. Comparison of periods obtained by linear and non-linear theories.

$\frac{P}{P_{0}}=\frac{\text { non-linear period }}{\text { linear period }}$;

$\frac{\Delta T_{\max }}{T_{0}}=\frac{\epsilon^{2}}{4}=\left(\frac{\text { umplitude }}{2 \alpha}\right)^{2}$.

Motion defined by Eqs. (15). $\alpha \neq 0$.

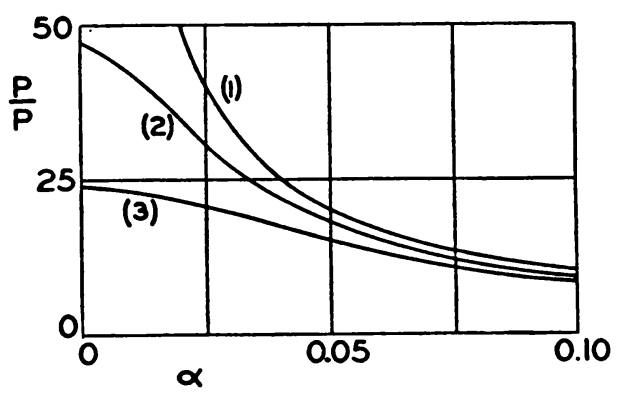

FIG. 3. Period v.s. initial tension.*

$$
\frac{P}{P^{*}}=\frac{\text { non-linear period }}{\left(E \pi^{2} / \rho l^{2}\right)^{1 / 2}} ; \quad \alpha^{2}=T_{0} / E A
$$

(1) vanishing amplitude (linear theory)

(2) $a=\alpha \epsilon=$ "amplitude" $=0.05$

(3) $a=0.10$

3. The perturbation procedure. It is convenient to choose, as the perturbation parameter of the problem, a number $\epsilon$ which is essentially the amplitude of the motion. ${ }^{* *}$ The two functions $\varphi$ and $\tau$ are therefore expanded in powers of this parameter as follows :

$$
\varphi=\alpha\left[\epsilon \varphi_{1}+\epsilon^{3} \varphi_{3}+\epsilon^{5} \varphi+\cdots\right], \quad \tau=\epsilon^{2} \tau_{2}+\epsilon^{4} \tau_{4}+\cdots .
$$

It is easily seen that a reversal of the sign of $\epsilon$ should merely reverse the sign of $\varphi$. Hence the omission of the even powers of $\epsilon$ is justified. In a similar manner the funtions $\tau_{1}, \tau_{3}, \ldots$ can be seen to vanish. That $\tau_{0}$ vanishes is seen by inspection of Eq. (4c). The expressions for $\varphi$ and $\tau$ are now substituted into Eqs. (4), the coeffi-

* In Fig 3. the ordinates should be labeled $P / P^{*}$.

** Equation (15) indicates more precisely the meaning of $\epsilon$. 
cients of each power of $\epsilon$ are equated to zero, and the following system of equations is obtained:

$$
\begin{array}{ll}
L_{0}\left(\varphi_{1}\right)=0, & (6 \mathrm{a}), \quad L_{1}\left(\tau_{2}\right)=-\alpha^{2} L_{0}\left(\frac{\varphi_{1}^{2}}{2}\right), \\
L_{0}\left(\varphi_{3}\right)=L_{1}\left(\tau_{2} \varphi_{1}\right), & (6 \mathrm{c}), \quad L_{1}\left(\tau_{4}\right)=-\alpha^{2} L_{0}\left(\varphi_{1} \varphi_{3}-\frac{\alpha^{2} \varphi_{1}^{4}}{8}\right), \\
L_{0}\left(\varphi_{8}\right)=L_{1}\left(\tau_{1} \varphi_{1}+\tau_{2} \varphi_{3}\right), & (6 \mathrm{e}), \quad L_{1}\left(\tau_{8}\right)=-\alpha^{2} L_{0}\left(\varphi_{1} \varphi_{5}+\frac{\varphi_{3}^{2}}{2}+\alpha^{2} \frac{\stackrel{\varphi}{1}_{1}^{3} \varphi_{3}}{2}+\alpha^{4} \frac{\varphi_{1}^{6}}{48}\right),
\end{array}
$$

where

and

$$
L_{0}=\frac{\partial^{2}}{\partial \xi^{2}}-\frac{\partial^{2}}{\partial \eta^{2}}, \quad L_{1}=\alpha^{2} \frac{\partial^{2}}{\partial \eta^{2}}-\frac{\partial^{2}}{\partial \xi^{2}}
$$

$$
\begin{array}{r}
\int_{0}^{\pi}\left(\tau_{2}-\frac{\varphi_{1}^{2}}{2}\right) d \xi=0 \\
\int_{0}^{\pi}\left(\tau_{4}-\varphi_{1} \varphi_{3}+\frac{\alpha^{2} \varphi_{1}^{4}}{8}\right) d \xi=0
\end{array}
$$

Since each of the operators in the foregoing equations is linear, it is now a simple matter to evaluate successively the $\varphi_{i}$ and the $\tau_{i}$. For the moment, we confine our attention to the motion defined by choosing as a solution to Eq. (6a) the function,

$$
\varphi_{1}=\cos \xi \cos \eta \text {. }
$$

Note that for $\varphi_{1}=\cos n \xi \cos n \eta$ the same solution will exist when $l$ is replaced by $l / n$ in the definitions of $\xi$ and $\eta$. Solving successively Eqs. (6), starting with the foregoing definition of $\varphi_{1}$, and using Eqs. (7) to determine the arbitrary terms appearing in the $\tau_{i}$, we obtain

$$
\begin{aligned}
\tau_{2}= & \frac{1}{4} \cos ^{2} \eta+\frac{\alpha^{2}}{8} \cos 2 \xi \\
\varphi_{3}= & \cos \xi\left[-\frac{3-2 \alpha^{2}-\alpha^{4}}{32} \eta \sin \eta+\frac{1-9 \alpha^{2}}{128} \cos 3 \eta-\frac{1}{128} \cos \eta\right] \\
& -\frac{\alpha^{2}\left(9-\alpha^{2}\right)}{128} \cos 3 \xi \cos \eta \\
\tau_{4}= & {\left[-\frac{3-2 \alpha^{2}-\alpha^{4}}{128} \eta \sin 2 \eta+\frac{1-9 \alpha^{2}}{512}(\cos 2 \eta+\cos 4 \eta)-\frac{3 \alpha^{2}}{512} \cos ^{4} \eta-\frac{1}{256} \cos ^{2} \eta\right] } \\
& +\frac{\alpha^{4}\left(21-\alpha^{2}\right)}{512} \cos 2 \xi+\cdots-\frac{3 \alpha^{2}}{2048} \frac{13-\alpha^{2}}{4-\alpha^{2}} \cos 4 \xi \cos 2 \eta
\end{aligned}
$$




$$
\begin{aligned}
\varphi_{5}= & \cos \xi\left[-\frac{9\left(1+\alpha^{2}+\cdots\right)}{2048} \eta^{2} \cos \eta-\frac{3}{512} \eta \sin \eta-\frac{9}{4096} \eta \sin 3 \eta+2^{-14} \cos 5 \eta\right] \\
& +\cdots,
\end{aligned}
$$

The arbitrary solutions of Eq. (6a) which may be added to each of the $\varphi_{i}$ as they are evaluated have been chosen in such a manner that $\lim \alpha \epsilon^{j} \varphi_{j}$ exists when $\alpha$ tends to zero and $\alpha \epsilon=$ constant $=\alpha$. ${ }^{*}$ This limiting process, of course, defines the motion wherein the initial tension $T_{0}$ is zero and the amplitude $a$ is non-vanishing. An investigation of this problem will simplify the question of the convergence of the functions $\varphi$ and $\tau$ as defined by Eqs. (5) and (8). When $\alpha$ tends to zero as specified above, the symbols $\tau$ and $\eta$ become meaningless. Hence, we replace them by

$$
\sigma=\frac{T}{E A}=\alpha^{2} \tau, \text { and } \eta=\alpha s .
$$

The limiting process then yields the following expressions for the $\varphi_{j}$ and the $\sigma_{j}$

$$
\begin{aligned}
& \alpha \in \varphi_{1}=a \cos \xi, \\
& \alpha \epsilon^{3} \varphi_{3}=a^{3}\left[-\frac{1}{2 !}\left(\frac{s}{2}\right)^{2} \cos \xi+\frac{9}{128} \cos \xi-\frac{9}{128} \cos 3 \xi\right], \\
& \alpha \epsilon^{5} \varphi_{5}=a^{5}\left[\frac{3}{4 !}\left(\frac{s}{2}\right)^{4} \cos \xi+\frac{5}{2^{8}}\left(\frac{s}{2}\right)^{2} \cos \xi-\frac{45}{128}\left(\frac{s}{2}\right)^{2} \cos 3 \xi+f(\xi)\right] \text {, } \\
& \alpha \epsilon^{7} \varphi_{7}=a^{7}\left[-\frac{27}{6 !}\left(\frac{s}{2}\right)^{6} \cos \xi+\cdots\right] \text {, } \\
& \epsilon^{2} \sigma_{2}=\frac{1}{4} a^{2}, \\
& \epsilon^{4} \sigma_{4}=a^{4}\left[-\frac{1}{16} s^{2}+\frac{\cos 2 \xi}{32}+\frac{37}{256}\right] \text {, } \\
& \epsilon^{6} \sigma_{\theta}=a^{6}\left[\frac{s^{4}}{128}-\frac{13 s^{2}}{512}+\frac{3 s^{2}}{128} \cos 2 \xi\right] \\
& +\left[\sigma_{4} \frac{\varphi_{1}^{2}}{2}+\sigma_{2} \varphi_{1} \varphi_{3}+\sigma_{2} \frac{\varphi_{1}^{4}}{8}\right] \alpha^{2} \epsilon^{6}+g(\xi), \\
& \epsilon^{8} \sigma_{8}=a^{8}\left[-\frac{s^{6}}{1280}+\cdots\right]
\end{aligned}
$$

These solutions may also be obtained, of course, by assuming $\alpha$ equal to zero at

* Such complementary solutions are usually chosen to be consistent with a given set of initial conditions. However, it is convenient here to choose them so that the solution does not become meaningless when $\alpha \rightarrow 0, \alpha \epsilon=a$. Equations (15) indicate that this choice leads to a solution corresponding to a nearly sinusoidal initial deformation. 
the outset, expanding $\varphi$ and $\sigma$ in powers of a parameter $a$, and proceeding in the foregoing manner.

Note that the leading terms of the $\varphi_{i}$ define an absolutely converging series for all $a$. Note also that the remaining terms of each $\varphi_{j}$ are dominated by this leading term. In fact, for sufficiently large $s$, the sum of the remaining terms in each $\varphi_{j}$ is as small as we please compared to this leading term. Although this dominance has not been shown to occur uniformly, it is to be expected that the series defined by Eqs. (9) and (10) will converge over some range of $a$. The requirement, "sufficiently large $s$ " introduces no difficulty since the initial value of $s$ may be chosen arbitrarily large.

The functions $\varphi$ and $\sigma$ are now most conveniently written in the forms

$$
\begin{aligned}
& \varphi(\xi, s, a)=a f_{1}(a s, \xi)+a^{3} f_{3}(a s, \xi)+\cdots, \\
& \sigma(\xi, s, a)=a^{2} g_{2}(a s, \xi)+a^{4} g_{4}(a s, \xi)+\cdots,
\end{aligned}
$$

where the terms of the series defining the $f_{i}$ and the $g_{i}$ are easily chosen from Eqs. (9) and (10). $f_{1}$ and $g_{2}$ are composed of the previously mentioned leading terms, and it is easily established that they converge to the values

$$
f_{1}=\mathrm{cn}\left(\frac{a s}{2}, \frac{1}{\sqrt{2}}\right) \cos \xi, \quad g_{2}=\frac{1}{4} \mathrm{cn}^{2}\left(\frac{a s}{2}, \frac{1}{\sqrt{2}}\right),
$$

where $c n$ denotes the elliptic cosine. Energy considerations may be used to show that the remaining $f_{j}$ and $g_{j}$ are bounded, and it is to be expected that the motion is closely described by $\varphi=a f_{1}$ and $\sigma=a^{2} g_{2}$ when $a$ is sufficiently small. For most materials, a value of $a^{2}$ greatly in excess of $10^{-3}$ will lead to plastic deformations; hence, the motion of such strings is well defined.

The motions arising when $T_{0}$ is arbitrary, as defined by Eqs. (5) and (8), can also be written in the form,

$$
\begin{aligned}
\varphi & =\alpha \epsilon F_{1}(\xi, \eta, \epsilon)+\alpha^{3} \epsilon^{3} F_{8}(\xi, \eta, \epsilon)+\cdots+P(\xi, \eta, \alpha, \epsilon), \\
\tau & =\epsilon^{2}\left[G_{2}(\xi, \eta, \epsilon)+\alpha^{2} \epsilon^{2} G_{4}(\xi, \eta, \epsilon+\cdots]+Q(\xi, \eta, \alpha, \epsilon),\right.
\end{aligned}
$$

where $P$ and $Q$ are those parts of $\varphi$ and $\tau$ which vanish when $\alpha$ tends to zero and $\alpha \epsilon=a$. For this case,

$$
F_{1}=\mathrm{cn}\left(\sqrt{1+\frac{\epsilon^{2}}{4}} \eta, k\right) \cos \xi, \quad G_{2}=\frac{1}{4} \mathrm{cn}\left(\sqrt{1+\frac{\epsilon^{2}}{4}} \eta, k\right),
$$

where $k=\epsilon\left[2\left(4+\epsilon^{2}\right)\right]^{-1 / 2}$. It is evident, in yiew of the foregoing results, that

$$
\lim _{\epsilon \rightarrow \infty} F_{j}(\xi, \eta, \epsilon)=f_{j}(\xi, a s)
$$

and it is to be concluded that since the. series defining the $F_{i}$ converge as $\epsilon$ tends to infinity, they will also converge for the smaller values of $\epsilon$. Both $\alpha$ and $\alpha \epsilon$ must be small because of elastic considerations, which indicates that $P$ and $Q$ will also exist. We conclude therefore that the motion of the string, whose "amplitude" $\alpha \epsilon$ is of the order of magnitude required by elastic considerations, is adequately defined by the leading terms of Eq. (13). That is, in the first approximation, 


$$
\begin{aligned}
& \varphi=\alpha \epsilon \operatorname{cn}\left[\sqrt{1+\frac{\epsilon}{4}} \eta, k\right] \cos \xi \\
& \tau=\frac{\epsilon^{2}}{4} \mathrm{cn}^{2}\left[\sqrt{1+\frac{\epsilon^{2}}{4}} \eta, k\right] .
\end{aligned}
$$

Figs. 2 and 3 compare the results of this analysis with those of the linear theory.

4. The motion following an arbitrary initial deformation. The motions derived in the preceding section are obviously those corresponding to initial sinusoidal deformations. If the perturbation procedure is again carried out, and if for $\varphi_{1}$ the function $\varphi_{1}=\sum_{j} b_{j} \cos j \xi \cos j \eta$ is selected, a solution will be obtained, the leading terms of which contain no powers of $\alpha$ greater than unity. The solution so obtained will correspond to an initial deformation, $\varphi_{1}(\xi, 0)=\sum_{j} b_{j} \cos j \xi$. This predominating part of the solution may, however, be obtained by a simpler, less rigorous, procedure which nevertheless leads to identical results. We merely expand $\left(1-\varphi^{2}\right)^{1 / 2}$ in the conventional power series and omit in Eqs. (4), $\varphi^{n+2}$ as compared to $\varphi^{n}$, and $\alpha^{2}$ as compared to 1 . We thus obtain as replacement for Eqs. (4)

$$
\begin{aligned}
& \frac{\partial^{2}}{\partial \xi^{2}}[(1+\tau) \varphi]=\frac{\partial^{2} \varphi}{\partial \eta^{2}} \\
& \frac{\partial^{2} \tau}{\partial \xi^{2}}=0, \text { hence } \tau=\tau(\eta) \\
& \int_{0}^{\pi}\left(\alpha^{2} \tau-\frac{\varphi^{2}}{2}\right) d \xi=0
\end{aligned}
$$

Finally the first of these becomes

$$
\left[1+\frac{\alpha^{-2}}{2 \pi} \int_{0}^{\pi} \varphi^{2}(\xi, \eta) d \xi\right] \frac{\partial^{2} \varphi}{\partial \xi^{2}}=\frac{\partial^{2} \varphi}{\partial \eta^{2}} .
$$

The solution corresponding to the initial conditions specified at the beginning of this section is found by considering that solution of the form $\varphi=\alpha \sum_{j} b_{j} \cos j \xi \psi_{j}(\eta)$, where $\psi_{j}(0)=1$ for each $j$.

Upon substitution of this function, Eq. (17) yields the following set of ordinary differential equations

$$
\psi_{n}^{\prime \prime}+n^{2} \psi_{n}\left[1+\frac{1}{4} \sum_{j} b_{j}^{2} \psi_{j}^{2}\right]=0 .
$$

These may be written in the conventional operational form

$$
\left(D^{2}+n^{2}\right) \psi_{n}=-\frac{n^{2}}{4} \psi_{n} \sum_{j} b_{j}^{2} \psi_{j}^{2}
$$

and standard integration procedure leads immediately to the integral equation

$$
\psi_{n}(\eta)=\cos n \eta-\frac{\eta}{4} \int_{0}^{\eta} \sin n(z-\eta) \psi_{n}(z) \sum_{j} b_{j}^{2} \psi_{j}^{2}(z) d z
$$


The method of successive approximations when applied to this equation will produce a converging sequence of solutions. This method is obviously preferable to the direct application of the perturbation method, once the equivalence of the results has been established, since no minor terms are carried along in the algebraic work, no complimentary solutions need be added as the integration proceeds, ${ }^{*}$ and the $\tau_{i}$ do not appear when the function $\varphi$ is evaluated.

It is of interest to note that when $b_{j} \equiv 0$ for $j \neq 1$, Eq. (20) assumes the form

$$
\psi_{1}=\cos \eta-\frac{b_{1}^{2}}{4} \int_{0}^{\eta} \sin (z-\eta) \psi_{1}^{3}(z) d z
$$

and that this equation must generate the elliptic function previously encountered. When the method of successive approximations is applied to this equation, the series obtained is that one found in the first solution obtained in this paper. This function may be obtained more directly by solving Eq. (18) for this particular set of initial conditions.

Perhaps the quickest way to obtain an approximation to the motion for nonsinusoidal initial deformation is to be found in the application of a numerical procedure using finite differences. Equation (17) lends itself readily to such a treatment and the results are considerably easier to interpret than those found by the more rigorous integral equation treatment.

5. The three dimensional problem. If we now allow deflections $w$ normal to the plane of $u$, the procedure of the foregoing sections of this paper leads to the equation

$$
\left\{1+\frac{\alpha^{-2}}{2 \pi} \int_{0}^{\pi}\left[\varphi^{2}(\xi, \eta)+\chi^{2}(\xi, \eta)\right] d \xi\right\} \frac{\partial^{2} \varphi}{\partial \xi^{2}}=\frac{\partial^{2} \varphi}{\partial \eta^{2}}
$$

and to the equation obtained by interchanging $\varphi$ and $\chi$ in (22). $\tau$ is given by the integral on the left side of this equation and $\chi=w /\left(1+\alpha^{2} \tau\right)$. It follows immediately from the similarity of Eq. (17) and that given above that the integral equation method previously described will provide the solutions to problems of this nature. In particular, however, the motions wherein the string at any instant lies in a single plane and wherein each particle describes a quasi-elliptical path is easily determined in closed form by considering the deformation expressed in the complex form

$$
\varphi=\epsilon \alpha \psi(\eta) e^{i \psi(\eta)} \cos \xi,
$$

where $\psi$ and $\mu$ are each real. Equation (22) assumes the form,

$$
\left[1+\frac{\epsilon^{2}}{2} \int_{0}^{\pi}|\varphi(\xi, \eta)|^{2} d \xi\right] \frac{\partial^{2} \varphi}{\partial \xi^{2}}=\frac{\partial^{2} \varphi}{\partial \eta^{2}}
$$

which, when separated into its real and imaginary parts, implies,

$$
\mu^{\prime}(\eta)=c / \psi^{2}(\eta)
$$

* When dealing with the differential equations leading to Eq. (8), it was necessary to choose complementary solutions to conform to given initial (or other auxiliary) conditions of the problem. In the integral equation approach, such conditions are always included in the equations. 
and

$$
\psi^{\prime \prime}+\psi+\frac{\epsilon^{2}}{4} \psi^{3}-c^{2} \psi^{-3}=0
$$

Here, $c$ is a constant defined by the initial conditions as follows;

$$
\psi(0)=1, \quad \psi^{\prime}(0)=0, \quad \mu(0)=0, \quad \mu^{\prime}(0)=c .
$$

When $c<1+\epsilon^{2} / 4$, these initial conditions lead to a solution of Eq. (24) given by

$$
\begin{aligned}
& \psi=\left[1-(1-\gamma) \operatorname{sn}^{2}\left\{\sqrt{\frac{1+\beta}{8}} \epsilon \eta, \sqrt{\frac{1-\gamma}{1+\beta}}\right\}\right]^{1 / 2} \\
& \mu=c \int_{0}^{\eta} \psi^{-2}(s) d s, \quad \tau=\frac{\epsilon^{2}}{4} \psi^{2},
\end{aligned}
$$

where

$$
\begin{aligned}
& \beta=\frac{1}{2 \epsilon^{2}}\left[\sqrt{\left(8+\epsilon^{2}\right)^{2}+32 \epsilon^{2} c^{2}}+\left(8+\epsilon^{2}\right)\right], \\
& \gamma=\frac{1}{2 \epsilon^{2}}\left[\sqrt{\left(8+\epsilon^{2}\right)^{2}+32 \epsilon^{2} c^{2}}-\left(8+\epsilon^{2}\right)\right] .
\end{aligned}
$$

Note that as $c$ tends to $1+\epsilon^{2} / 4, \psi$ becomes identically unity and the motion of each particle is circular. That is,

$$
\varphi=\alpha \epsilon \cos \xi e^{i \eta \sqrt{1+\epsilon / 4}} .
$$

When $c>1+\epsilon^{2} / 4$, integration of Eq. (24) yields,

$$
\psi^{2}-1=\frac{(\beta+1)(\gamma-1) Z^{2}}{\gamma+\beta-(\gamma-1) Z^{2}}
$$

where

$$
Z=\operatorname{sn}\left[\sqrt{\frac{\gamma+\beta}{8}} \epsilon \eta, \sqrt{\frac{\gamma-1}{\gamma+\beta}}\right] .
$$

It is interesting to observe that the string never passes through its rest position for values of $c$ different from zero. This follows from the fact that $\psi$ never vanishes.

The function which rigorously defines the transmission of a localized disturbance along the string is easily found by considering those solutions of Eqs. (4) which allow the function $\tau$ to assume a constant value. Equations (4) become, under this assumption,

$$
p^{2} \frac{\partial^{2} u^{\prime}}{\partial \xi^{2}}=\frac{\partial^{2} u^{\prime}}{\partial \eta^{2}}, \quad p^{2} \frac{\partial^{2} v^{\prime}}{\partial \xi^{2}}=\frac{\partial^{2} v^{\prime}}{\partial \eta^{2}}, \quad \int_{0}^{\pi}\left[\left(1+\alpha^{2} \tau\right)^{2}-\left(u^{\prime}\right)^{2}\right]^{1 / 2} d \xi=\pi,
$$

where

$$
p^{2}=\frac{1+\tau}{1+\alpha^{2} \tau}
$$


If we now choose

$$
u^{\prime}=f(\xi-p \eta), \quad v^{\prime}=\left\{\left(1+\alpha^{2} \tau\right)^{2}-\left(u^{\prime}\right)^{2}\right\}^{1 / 2}-1,
$$

where $\tau$ is determined by

$$
\frac{1}{\pi} \int_{0}^{\pi}\left\{\left[1+\alpha^{2} \tau\right]^{2}-\left|u^{\prime}(\xi, 0)\right|^{2}\right\}^{1 / 2} d \xi=1,
$$

and where $f(\xi)$ is non-vanishing in a small region in $\xi$, all equations are satisfied. This solution is valid until the deformation reaches a fixed point in the string. When this occurs, the reflection phenomenon requires a change in $\tau$. This solution is in agreement with that found by the linear theory except that $p$ would assume the value unity in that theory. 